\author{
EVS29 Symposium \\ Montréal, Québec, Canada, June 19-22, 2016
}

\title{
Residential Smart-Charging Pilot Program in Toronto: Results of a Utility Controlled Charging Pilot
}

\author{
J. Bauman ${ }^{1}$, M.B. Stevens ${ }^{1 *}$, S. Hacikyan ${ }^{1}$, L. Tremblay ${ }^{2}$, E. Mallia ${ }^{1}$, C.J. Mendes ${ }^{1}$ \\ ${ }^{I}$ Matthew Stevens, FleetCarma (division of CrossChasm Technologies Inc.), 2-60 Northland Rd. Waterloo (Ontario) \\ Canada N2V2B8, mstevens@fleetcarma.com
}

${ }^{2}$ AddÉnergie Technologies Inc., 2327 Versant Nord Blvd, suite 120, Québec (Québec), Canada G1N 4C2

\begin{abstract}
Summary
Thirty plug-in electric vehicle (PEV) owners in Toronto participated in the 15-month ChargeTO program, which actively curtailed their vehicles during charging. The intent was to demonstrate the technical feasibility of the smart-charging system and evaluate its limitations, quantify the real-world curtailment availability of the PEVs, and to capture the participant's impressions and response to various incentive structures. A key feature of this program was the use of vehicle-side data, namely battery state-of-charge (SOC), to ensure that charge curtailments did not negatively affect the participants. This paper summarizes the findings from the ChargeTO program.
\end{abstract}

Keywords: load management, smart grid, utility, charging, demonstration

\section{Introduction}

The number of light-duty plug-in electric vehicles (PEVs) on the roads worldwide surpassed 1 million in September 2015 [1], and analysts are now forecasting 100 million PEVs on the road worldwide by 2030 [2]. The large and increasing numbers of PEVs on the road are causing valid concerns over grid reliability and infrastructure upgrade costs required to meet peak demands - some networks are already grappling with these issues today [3],[4]. In theory, utility controlled charging (UCC) or smart-charging (moderating PEV charging via network control) has the potential to address these issues, and more [5]. The benefits of smartcharging are well understood, and include: increasing grid reliability, lowering generation costs and carbon intensity, lowering upgrade costs for grid infrastructure, and coupling PEV charging loads with generation from renewables. Despite these potential financial and environmental benefits, smart-charging has not yet been deployed at any significant scale using a variety of vehicle types. This lack of progress is due to the following factors: (i) PEV drivers have indicated they prefer to enroll in smart-charging programs that protect them from charge curtailment at low battery charge [6], which requires vehicle-side data including battery state-of-charge (SOC), and (ii) the availability of the required vehicle-side data has not been standardized for production PEVs for use in a smart-charging programs. Multiple, and potentially competing, standards are under development to resolve this for future production vehicles. In advance of vehicles complying with those standards there is a need to deploy smart-charging systems to resolve real-world deployment issues, enable program development, and to understand the potential acceptance of PEV owners to these programs.

The most recent real-world demonstrations of smart-charging generally fall into one of 3 categories: (i) small controlled tests with a limited number of vehicles [7], [8], (ii) larger scale demonstrations that do not use vehicle side data [9], [10], and (iii) larger scale demonstrations that use vehicle-side data but only include one vehicle model because the method of obtaining the vehicle data is unique to that vehicle model [11], 
[12], [13], [14]. In [9], ElaadNL, the innovation center for charging infrastructure in the Netherlands, is creating a large-scale smart-charging pilot. However, since vehicle-side data is not currently available in this pilot, the strategy is to reduce charging power between $5 \mathrm{pm}$ to $7 \mathrm{pm}$ each day, regardless of battery SOC. In [10], Southern California Edison (SCE) is testing out demand response for workplace charging with 80 networked EVSEs for employee use at SCE campuses. Since there is no vehicle-side data, drivers can opt-in or opt-out of each DR event using an app.

The value of using vehicle-side data to optimize charge scheduling is generally recognized, and thus numerous projects are developing this type of smart-charging system for a single vehicle model. In [11], five Duke Energy employees and five Duke Energy customers trialled a smart-charging system using Toyota Plug-In Priuses. The Priuses were configured to send vehicle-side data to a Toyota Smart Center cloud-based server. In [12], My Electric Avenue in the UK ran a project where participants drove 100 Nissan Leafs and participated in smart-charging - the Nissan Carwings system was used to report vehicle data back to a central server. References [13] and [14] highlight projects that are currently in progress, and although there are no final project reports available at this time, they help to illustrate some of the most recent work in this field. The second phase of the Jump Smart Maui project in [13] is looking to enroll 300 participants who drive Nissan Leafs in Maui who will participate in smart-charging - again, the Nissan Carwings system will be used to report vehicle data back to a central server. In [14], PG\&E and BMW are working together in the BMW i Chargeforward program to control charging on 100 BMW i3s in the San Francisco Bay Area - the BMW i3 communication system will be used to report vehicle data to a main server. These projects all have a common theme: vehicle-side data is seen as valuable in balancing the needs of the grid with the needs of the driver and so is a required part of the project, yet only single vehicle models can be used because the programs are not using technology to aggregate vehicle-side data from a variety of EV models, which is a necessary component of creating wide-scale commercial smart-charging systems that use vehicle-side data.

FleetCarma's connected car platform for electric vehicles, while not originally designed for smart-charging, can play an enabling role in smart-charging. The system is used by fleets and individuals in 23 countries to support the real-world operations of their electric vehicles. The relevant component of FleetCarma's system for smart-charging is its ability to log vehicle-side data, including battery SOC, on over 45 different PEV models. As a result, FleetCarma's system is capable of providing the necessary vehicle-side data to do large smart-charging deployments with many different plug-in models. As a result FleetCarma created a smartcharging system to work with the data from the FleetCarma logger, and has now successfully demonstrated this smart-charging system in the ChargeTO Pilot, which ran from October 2014 to January 2016. The ChargeTO Pilot is the world's first residential smart-charging demonstration that uses vehicle-side data from multiple PEV models. This paper reports on the results and learnings from this demonstration.

The ChargeTO project, run in collaboration with the local utility, began in late 2014 with the recruitment and enrollment of 30 participants who drove PEVs in the Toronto area. The participants were incentivized with a free AddÉnergie Core+ Level 2 EVSE (electric vehicle supply equipment) and free use of the FleetCarma vehicle logger with fleet-grade vehicle monitoring during the project. At the completion of the project, participants retained full ownership of their new EVSE, and had the option of trading in the cellular fleetgrade vehicle logger for a Bluetooth personal vehicle logger. Interest from the public was high, as the program had over 100 people register for the 30 spots. EVSE and vehicle logger installations occurred in Winter 2015, along with baseline data collection of charging and driving behavior. Curtailment testing began in Spring 2015. By mid-Summer 2015, participants were being regularly curtailed, and an incentive phase occurred in October and November 2015. Curtailment ended and data analysis began in January 2016. The main goals of the project were:

1. Test and refine the smart-charging system with real-world data to prepare for commercialization.

2. Obtain charging and user preferences data to quantify charge shift possibilities.

3. Obtain feedback from participants to investigate the potential requirements for engaging PEV owners in a wide-spread smart-charging program.

\section{FleetCarma Smart-Charging System}

The FleetCarma smart-charging system includes four main components, which are shown in Figure 1.

1. FleetCarma Charge Management Controller: cloud-based algorithm which uses inputs from the utility (maximum power request), driver (smart-charging preferences), and vehicle to calculate the optimal charge curtailment schedule to best satisfy both the utility and driver needs. 
2. FleetCarma Vehicle Logger: plugs into the on-board diagnostics port in each vehicle to gather data (battery SOC, charging power, GPS coordinates, etc.).

3. FleetCarma Smart-Charging Portal: responsive web portal where drivers enter smart-charging preferences.

4. Control Pathway: any control pathway that allows the FleetCarma Charge Management Controller to actually curtail charge at the EVSE. In this project, two control pathways were used: (i) the networked AddÉnergie Core+ Level 2 EVSE, and (ii) the Tesla API, which was preferred by some Tesla Model S owners who chose to keep their high-powered Tesla chargers and gave FleetCarma permission to control their charging through the Tesla API.

Note that the Utility Command Signal can also be generalized to come from any entity that is responsible for controlling the total charging power. For example, this maximum power request could be set by a facility or fleet manager who is responsible for curtailing EV charging during demand response (DR) events, or adhering to a daily limit to reduce facility demand charges.

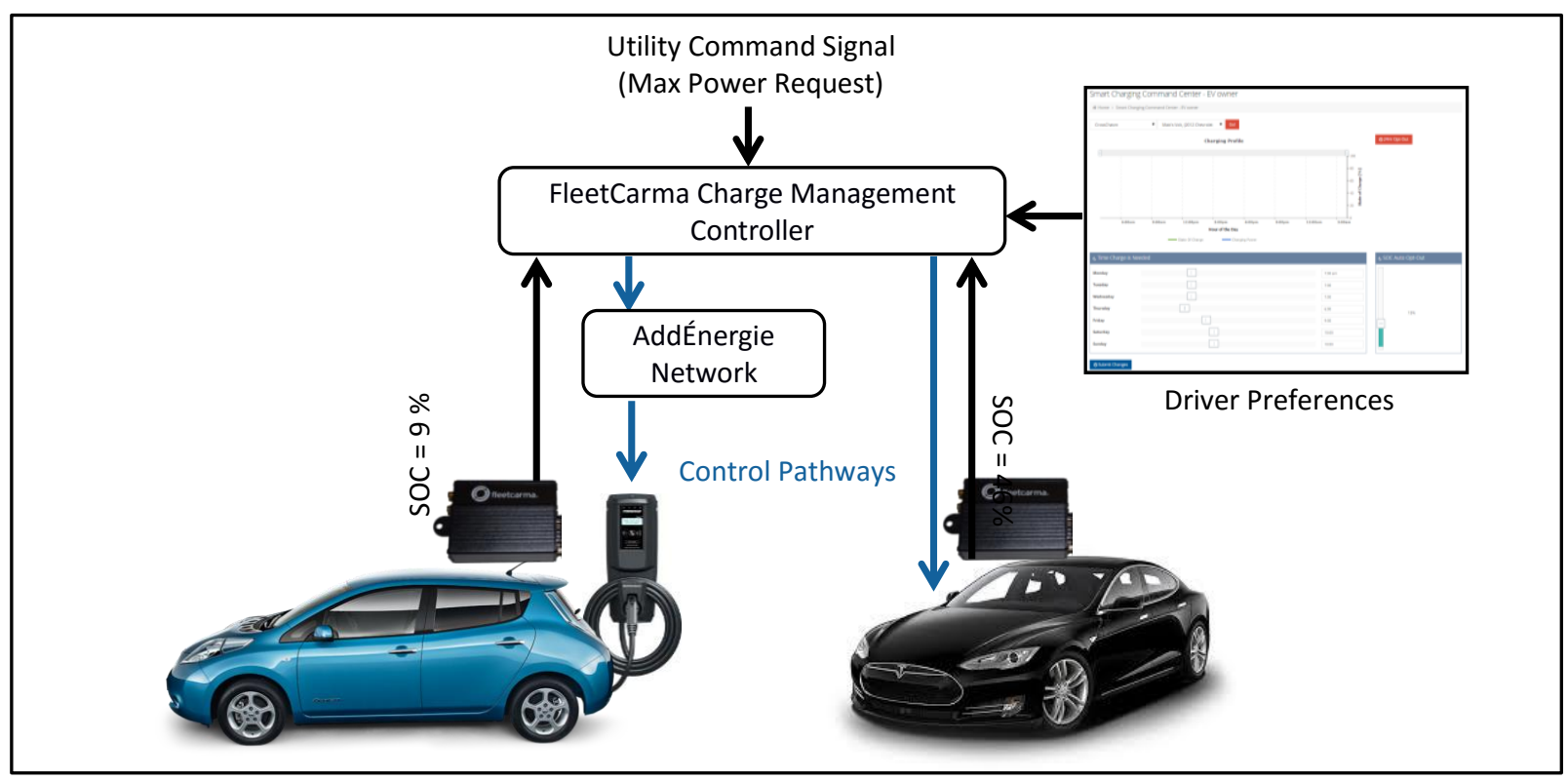

Figure 1: FleetCarma Smart-Charging System (ChargeTO Pilot Configuration)

\subsection{FleetCarma Smart-Charging Portal}

The FleetCarma Smart-Charging Portal has two main views: (i) the individual EV owner view and (ii) the utility fleet charging view. The EV owner view is shown in Figure 2. There are 3 control inputs that the driver can set on this page. The highlighted box 1 indicates the $24 \mathrm{Hr}$ Opt-Out button. In the ChargeTO pilot, the driver was allowed to hit this button as many times as needed, and would be unavailable for curtailment by the system for the 24 hours following each button press. The highlighted box 2 indicates the Time Charge Is Needed (TCIN) setting. Here the driver sets the time for each day of the week when they need to have their battery fully charged. The algorithm ensures that any curtailment performed before this time will allow a large enough charging window for the vehicle to reach full charge by TCIN, given the vehicle's current battery SOC. The highlighted box 3 indicates the SOC Auto Opt-Out setting. This setting allows the driver to set a minimum SOC, such that their vehicle is not curtailed if their current SOC is below this setting (effectively, they have a protected minimum SOC before they would be available for curtailment). For the ChargeTO pilot, this setting was allowed to range from $0 \%$ to $50 \%$ of battery SOC. The Charging Profile plot displays the real-time battery SOC and charging data to the driver.

The fleet charging view (Figure 3) provides the control input to the utility (or fleet manager, facilities manager, etc.) to control the maximum charging load of the PEV fleet. This control is currently performed by adjusting the slide bar in the highlighted box, Auto-Curtailment Threshold. FleetCarma is currently in the process of becoming OpenADR certified, such that this control can be provided automatically from the utility. In the example in Figure 3, the Auto-Curtailment Threshold was set to $10 \mathrm{~kW}$ for the fleet consisting of the ChargeTO participants. The Daily Load Profile plot shows the aggregated load of the fleet over the last day. The red line shows what the fleet charging power would have been if there was no curtailment. The dark blue 
line indicates what the actual charging power was, which can be seen to be limited to approximately $10 \mathrm{~kW}$. The light blue line shows the minimum charging power that would have been required to fulfill all driver requirements, as set by the 3 settings in the EV owner view. On this evening, there was a maximum of $27 \mathrm{~kW}$ of curtailment. A historical reporting feature is also available in the portal to show historical power and energy consumption when smart-charging was used, and to estimate what would have happened if smart-charging was not used.

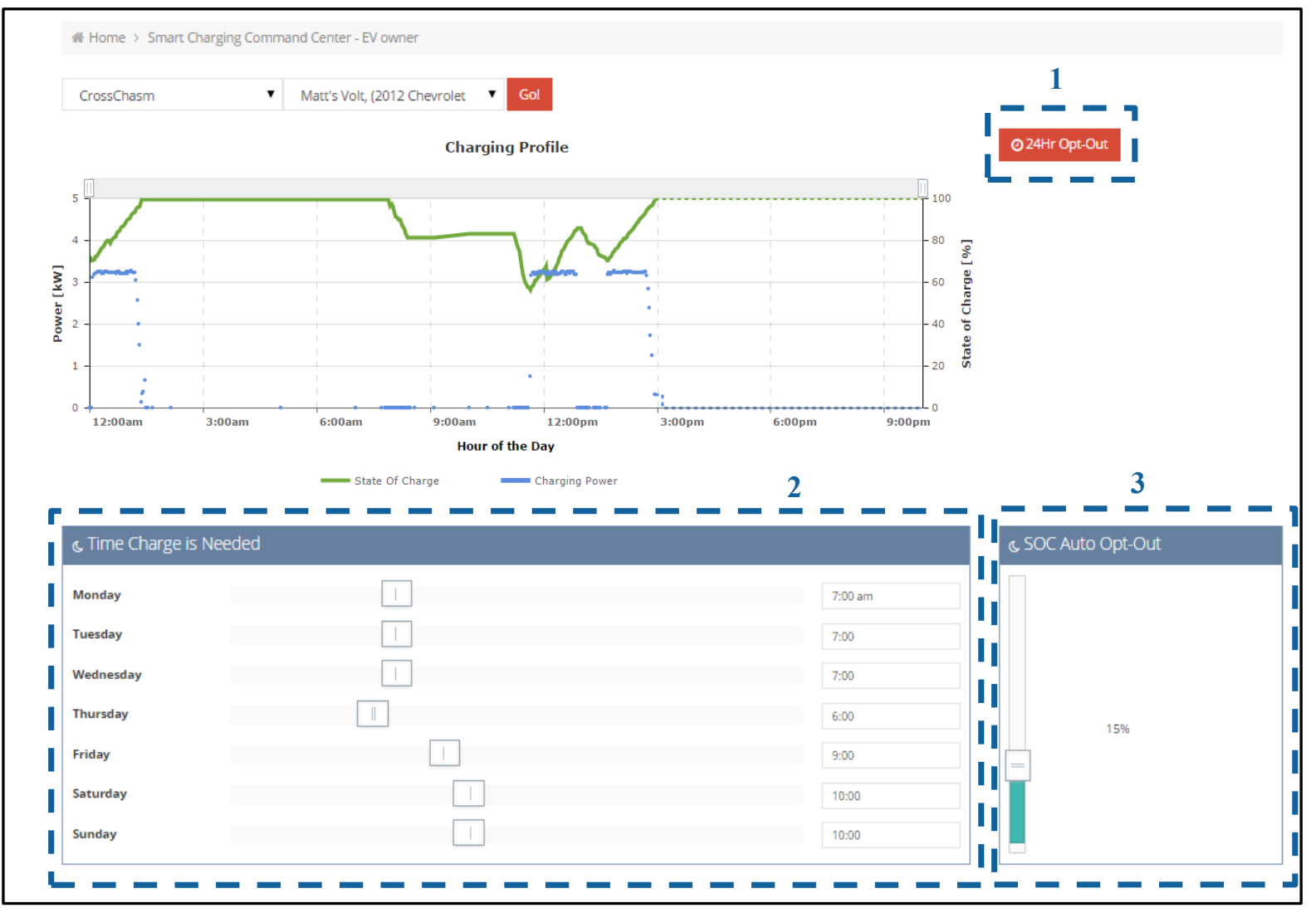

Figure 2: FleetCarma Smart-Charging Portal EV Owner View

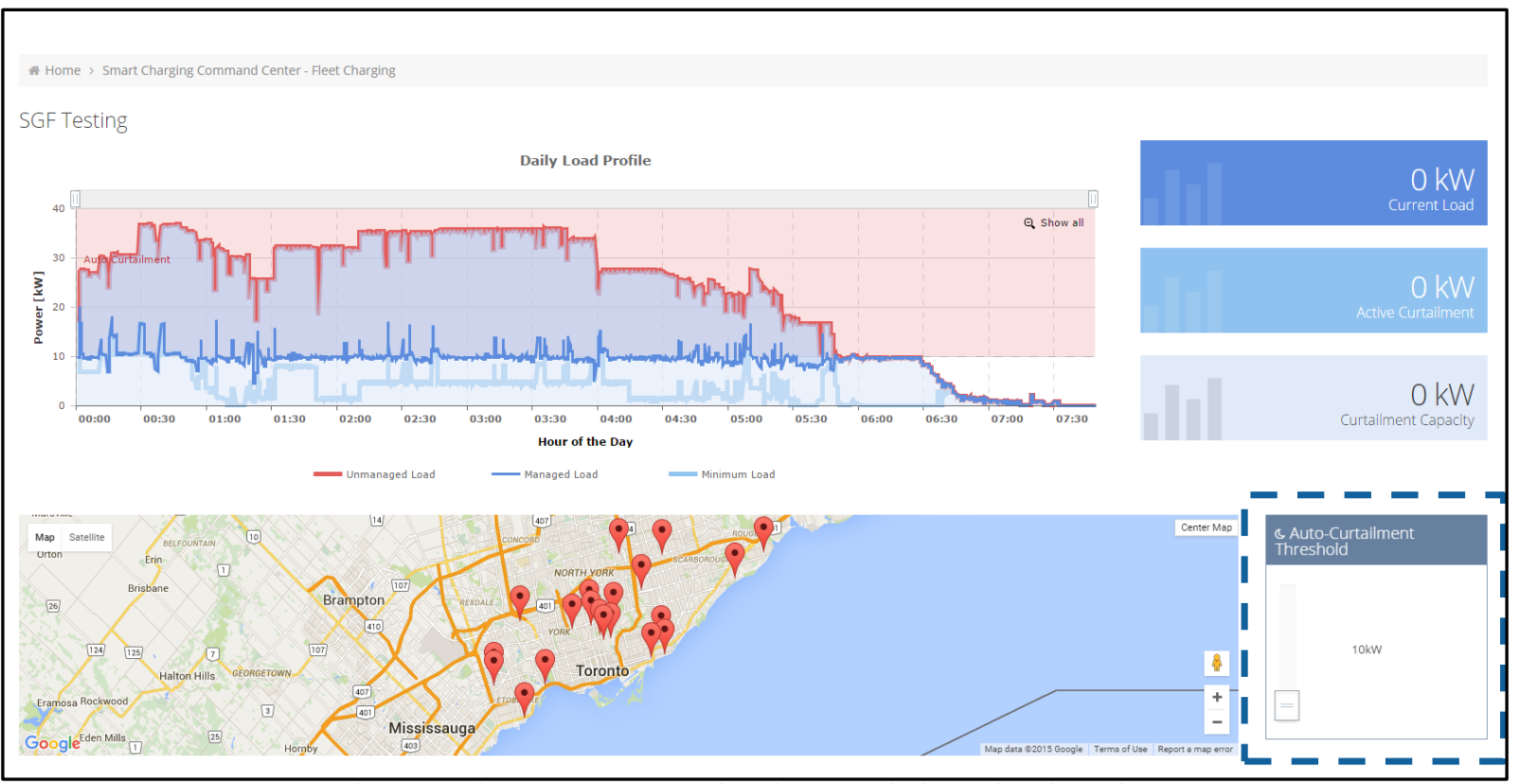

Figure 3: FleetCarma Smart-Charging Portal Fleet Charging View 


\section{Baseline Charging Results}

Baseline charging data was collected between February and June of 2015. This data shows how the participants charged their vehicles when no smart-charging occurred, including charging at any location (not just at home). Figure 4 shows the average charging power per vehicle on a weekday and Figure 5 shows the average charging power per vehicle on the weekend. The overall month-to-month trend in both of these figures shows that more charging power is required in the colder months (February and March) compared to the warmer months. This is to be expected because for the same average driving distance across all months, each PEV will use more energy in cold months due to electric heater use and battery inefficiencies in cold temperatures. On the weekday plot, there are 3 peak charging periods (early evening, overnight, midmorning), reflecting 3 categories of participant charging behavior:

1. People who charge in the early evening (peak starting around $7 \mathrm{pm}$ ). This could be due to simply plugging in when they return home for the day or setting an early evening timer for when Ontario Time of Use rates reduce (at $7 \mathrm{pm}$ ). $50 \%$ of the participants exhibited this behavior.

2. People who schedule charge for the late evening or early morning to shift their charging away from highdemand time periods. $36 \%$ of the participants exhibited this behavior.

3. People who schedule charge to occur before they leave in the morning to warm up the battery and slightly increase their battery range (peak around 7-8am). 14\% of the participants exhibited this behavior.

The weekday plot (Figure 4) also shows that there is a measurable amount of charging occurring during the middle of the day, around $0.2 \mathrm{~kW}$ per vehicle on average. In contrast, the weekend plot (Figure 5) shows a higher average charge rate in the afternoon $(0.4 \mathrm{~kW})$, similar early evening charging levels, and lower overnight charging levels compared to the weekday plot. Note that the charging spike around 5pm on the weekend plot is the result of a DC fast charge event.

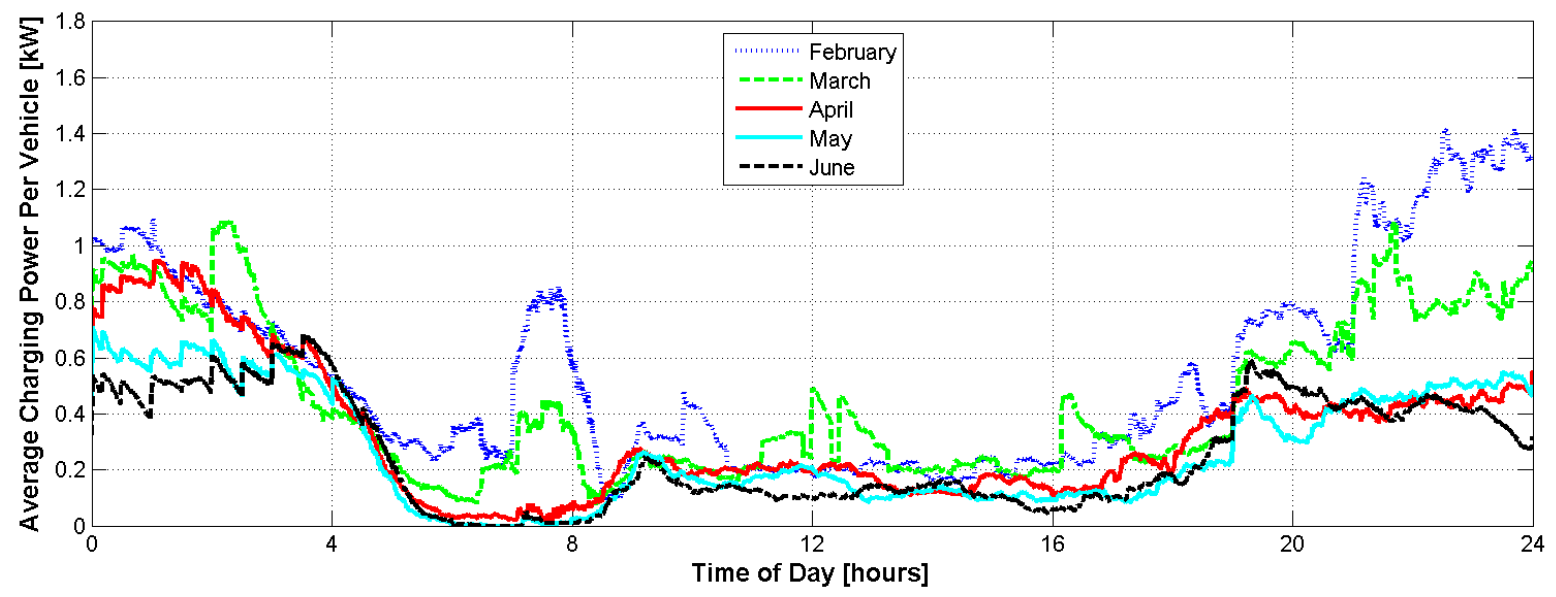

Figure 4: Baseline Charging For All Participants on a Weekday (Per Vehicle)

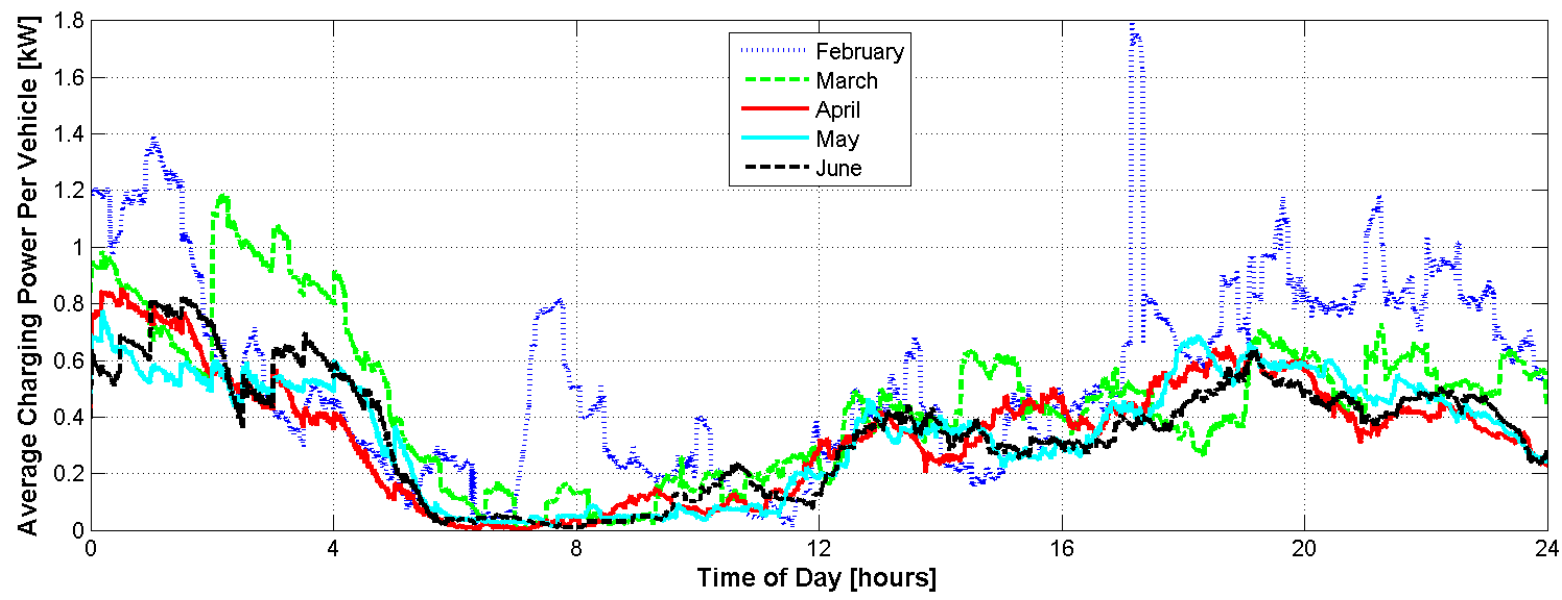

Figure 5: Baseline Charging For All Participants on the Weekend (Per Vehicle) 
The charging data analysis which follows seeks to quantify smart-charging benefits, both to the grid and for the environment, which can be extrapolated to the widespread adoption of PEVs and smart-charging. Since the ChargeTO pilot was composed of mainly early PEV adopters, it is not reasonable to assume the general public will follow the same charging patterns as these early adopters in terms of overnight charge scheduling. Thus, the analysis going forward will focus on the $50 \%$ of participants who charged without an overnight timer, and contributed to the early-evening peaks that are of the most concern to the grid and for the environment. Figure 6 shows the vehicle breakdown of all participants on the left and of this sub-group on the right. The corresponding baseline charging data for these $50 \%$ of the participants is shown in Figure 7 for the weekday and Figure 8 for the weekend. For this group, the peak charging power in the critical early evening time was $1.2 \mathrm{~kW}$ per vehicle at around $8 \mathrm{pm}$.
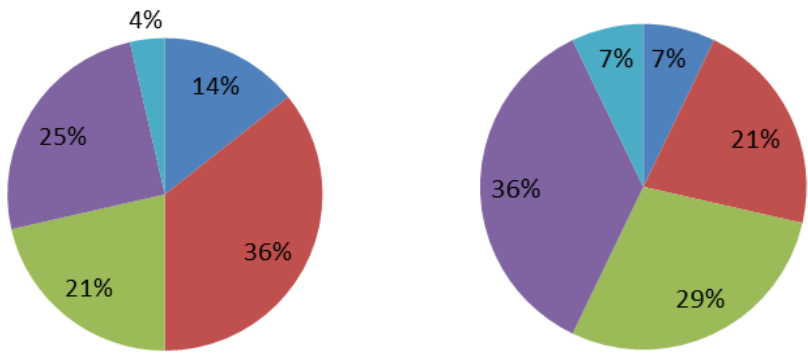

- Tesla Model S

Nissan Leaf

- Smart fortwo ED

Chevrolet Volt

BMW i3

Figure 6: PEV Model Breakdown For All Participants (left) and for 50\% of Participants Who Do Not Schedule Overnight Charging (right)

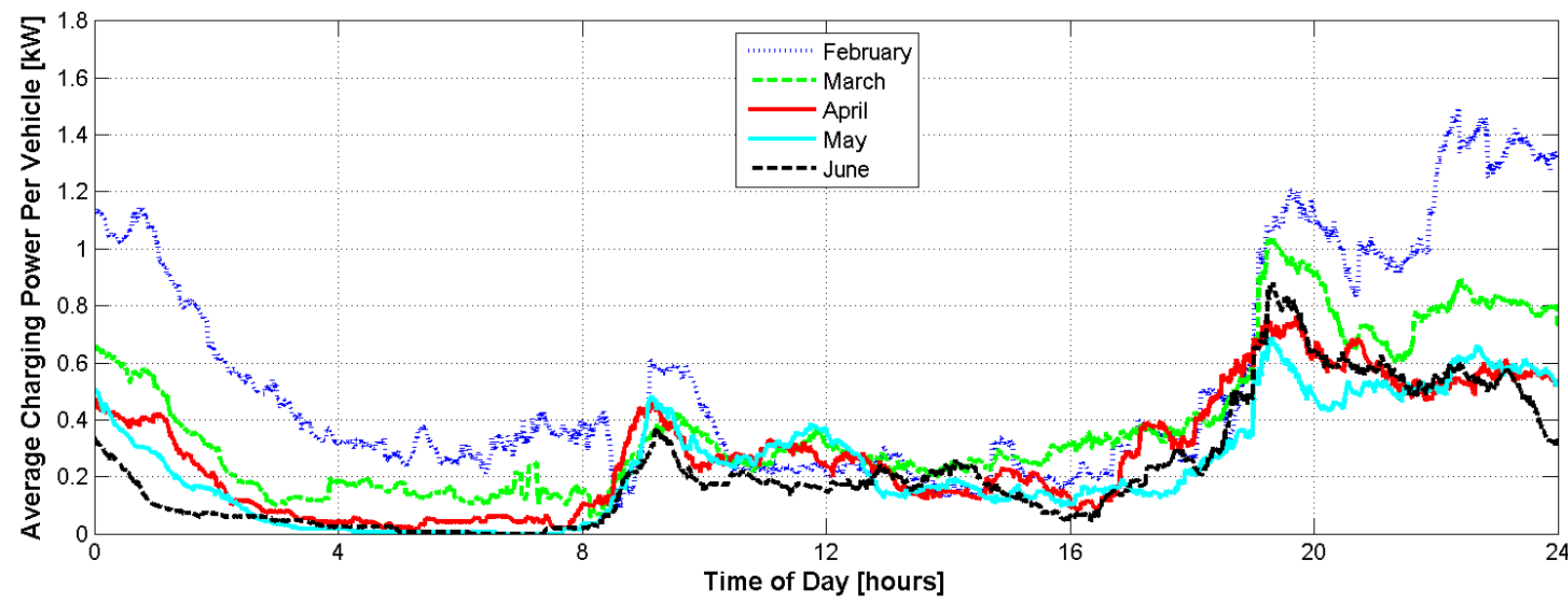

Figure 7: Baseline Weekday Charging For Participants Without Scheduled Overnight Charging (Per Vehicle)

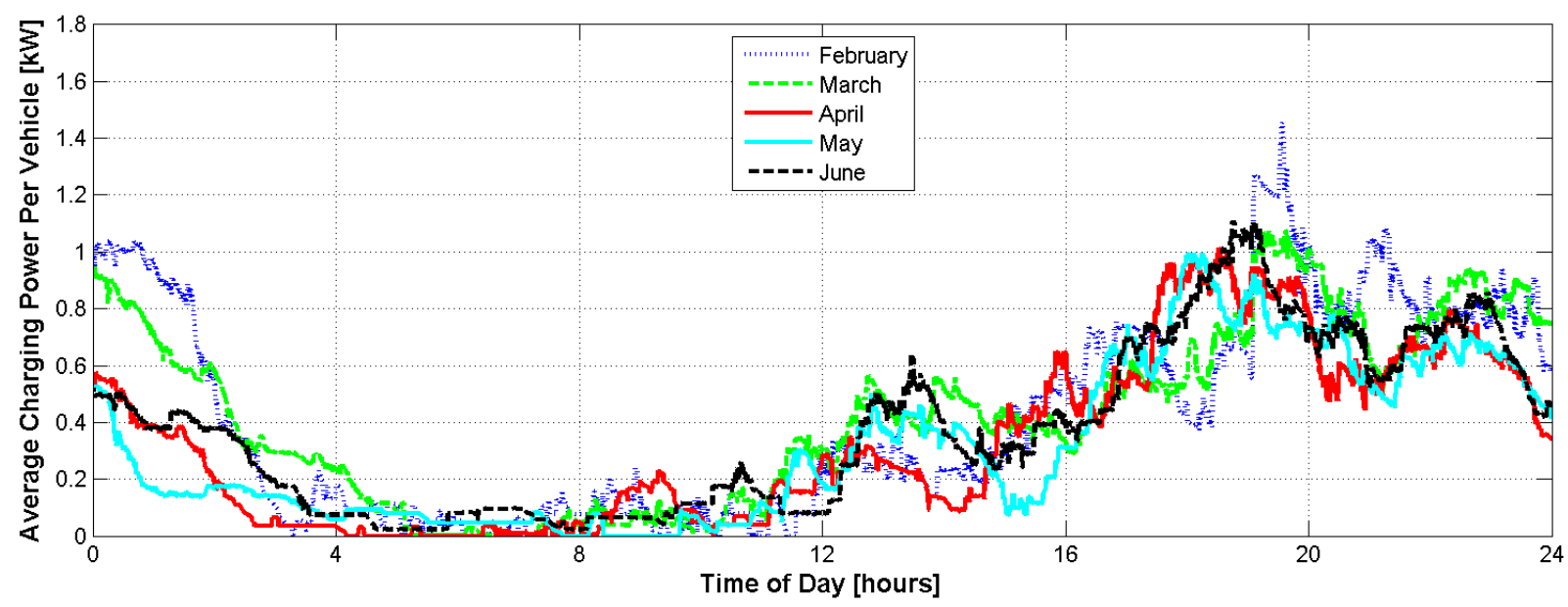

Figure 8: Baseline Weekend Charging For Participants Without Scheduled Overnight Charging (Per Vehicle) 


\section{Instantaneous Curtailment Results}

The data gathered in this program was used to calculate the average amount of instantaneous curtailment capacity available at any given time. This indicates how much load could be shed in the case of a grid emergency and when load can be routinely shifted to reduce peak loads on local infrastructure, possibly delaying the need for infrastructure upgrades. To calculate this instantaneous curtailment capacity the minimum load was subtracted from the unmanaged load. The minimum load is the sum of i) the charging power of vehicles that must be continuously charging at full power in order to be fully charged by TCIN, and ii) the charging power of any vehicles that are currently opted-out due to the PEV owner pressing the $24 \mathrm{Hr}$ Opt-Out button. Figure 9 shows the average per-vehicle instantaneous curtailment capacity on a weekday from August to December 2015, and Figure 10 shows the same for weekends. For example, in December, there was an average of $1.2 \mathrm{~kW}$ per vehicle curtailment capacity at $9 \mathrm{pm}$. This means that for a pool of 1000 smart-charging vehicles, the instantaneous load could be reduced by approximately $1.2 \mathrm{MW}$ at $9 \mathrm{pm}$.

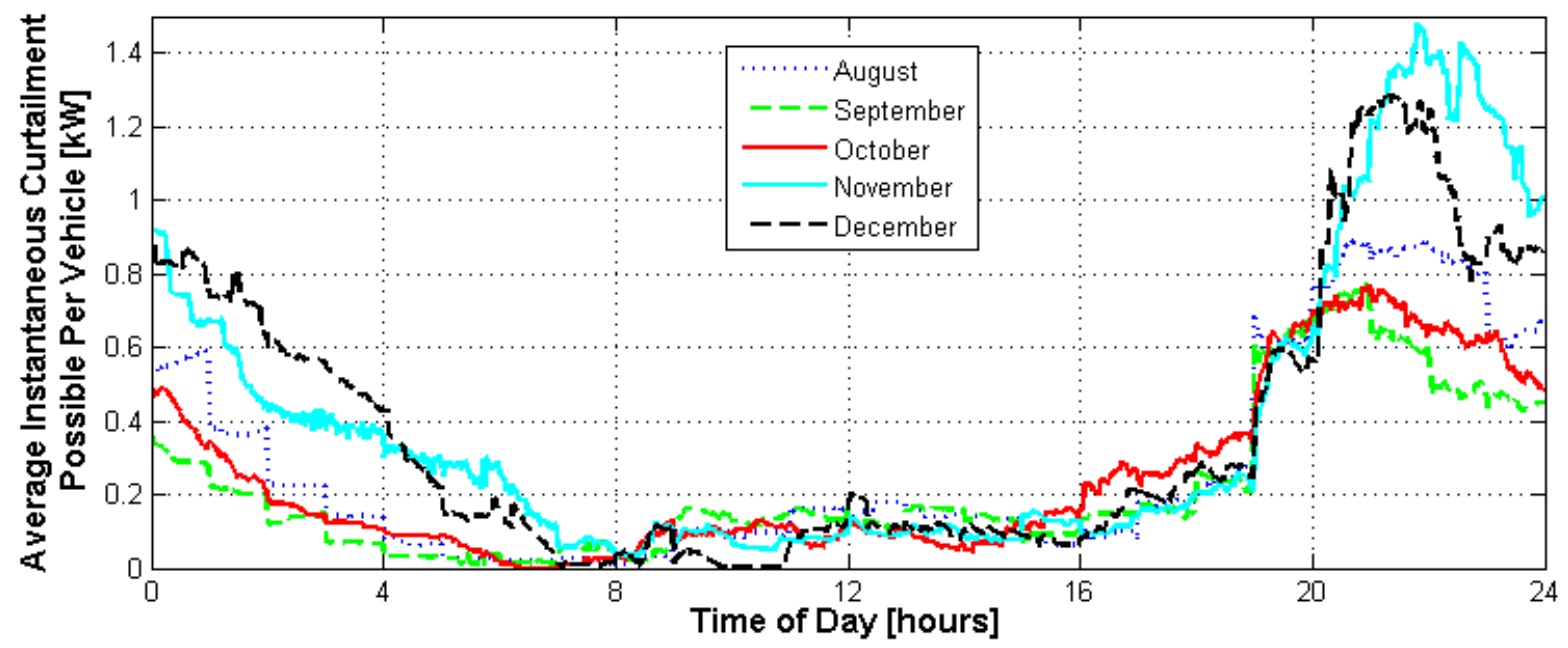

Figure 9: Average Per-Vehicle Instantaneous Curtailment Capacity on a Weekday

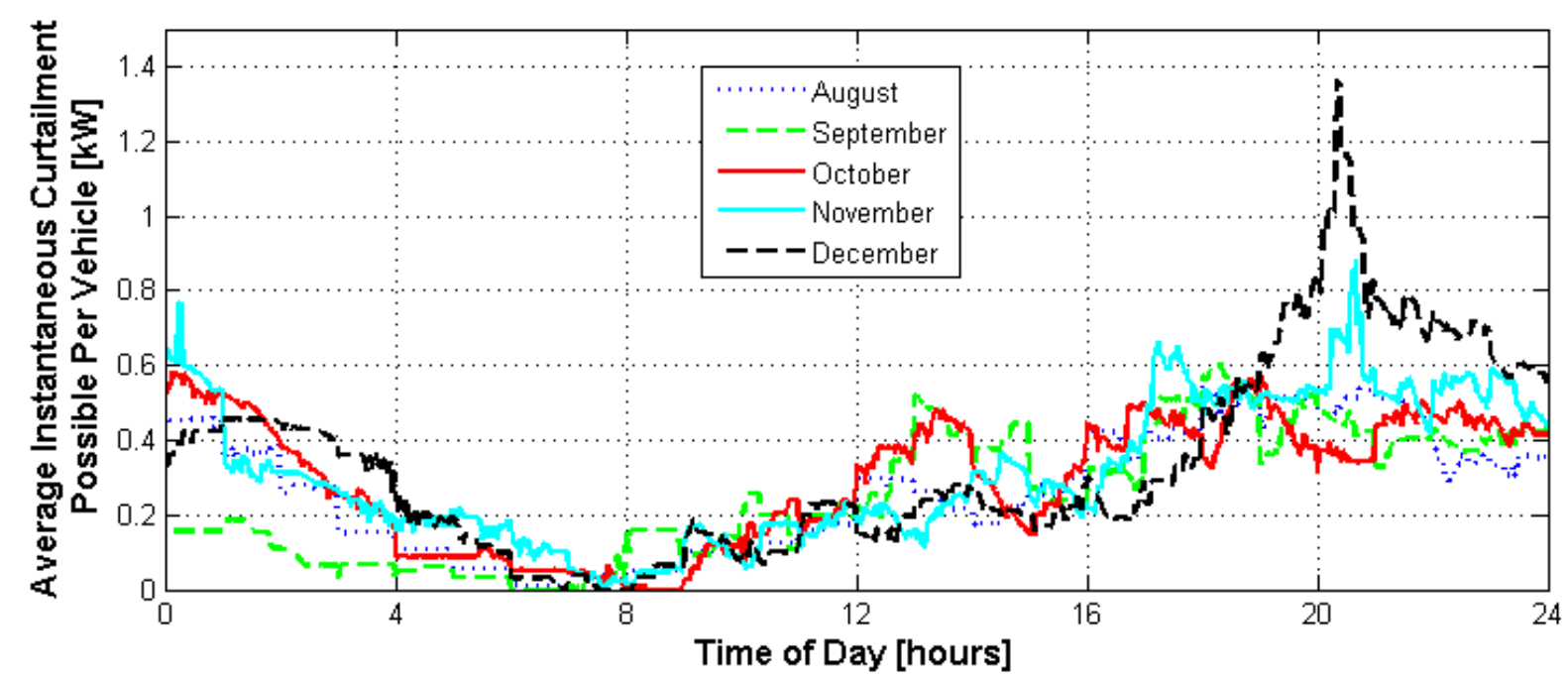

Figure 10: Average Per-Vehicle Instantaneous Curtailment Capacity on a Weekend

\section{Environmental Benefits}

The optimal way to shift PEV charging loads in order to maximize environmental benefits will vary by region, by season, and by day. Thus, it is important to have a centralized smart-charging system in place that can respond to local and seasonal grid conditions. This paper will discuss two examples of how different PEV load shift strategies can reduce grid emissions. 


\subsection{Shift Load To Improve Over-Generation of Renewables (i.e. Duck Curve)}

The duck curve, originally identified in [15], and further analyzed in [16], illustrates the concern of potential overgeneration and solar curtailment in areas of high photovoltaic (PV) penetration (such as California) due to the high generation of solar energy during midday. These concerns focus on sunny months that do not have high daytime air-conditioning loads, such as March and April. The duck curve is the net daily load in a high PV area, which is the normal load minus wind and PV generation. As can be seen in Figure 11, a large rampup of about 13,000 MW is needed over 3 hours to satisfy load in the late afternoon and early evening as the sun sets on a typical day in California. Since many of these resources need time to come on line before they can support these ramps, and also need to provide a minimal power level during this time, there is valid concern that PV generation may have to be curtailed during midday in the near future. This action would result in stranded financial resources of PV arrays being pulled offline, and also increase emissions as a fuelburning plant would be replacing power that could have been generated by existing PV arrays. A widespread smart-charging program can intelligently shift load away from the evening ramp towards midday to ease this problem. This type of PEV charging control could maximize the use of PV energy when it is available, and minimize the need to take these resources offline.

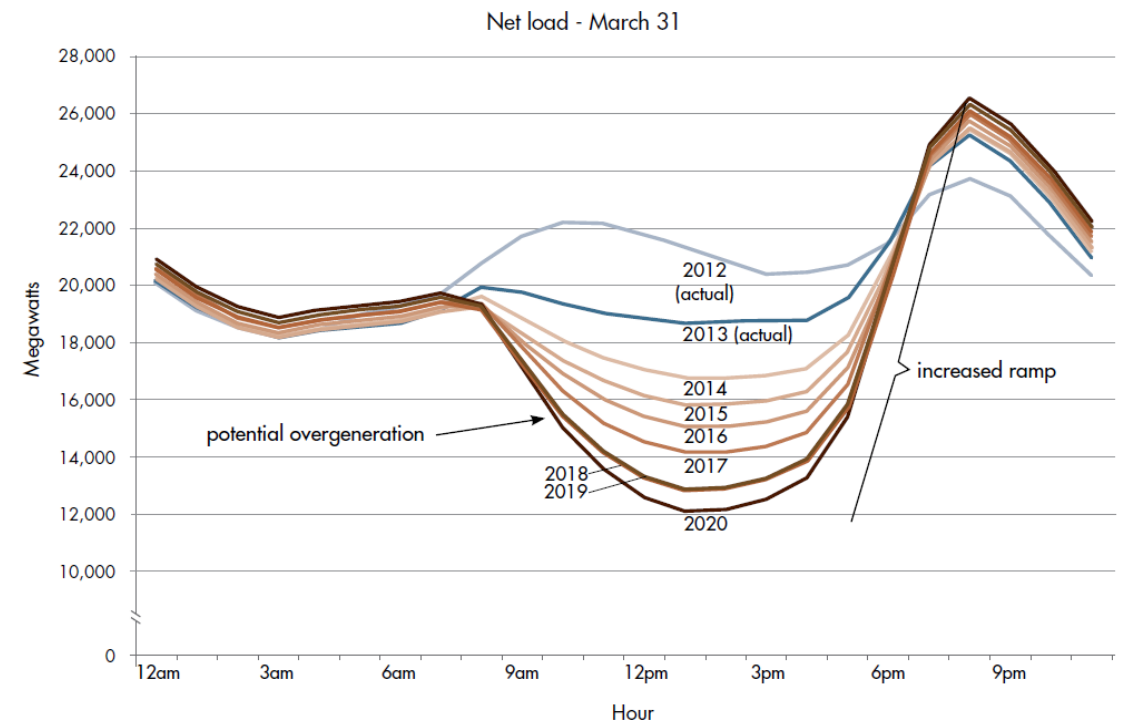

Figure 11: Duck Curve [15]

\subsection{Shift Load To Minimize Grid Emissions}

At times when the duck curve is not a concern, it is often desirable to shift load away from midday and early evening, as this is when load is generally highest and thus the most fuel-burning plants are operating. Though the optimal way to shift load will depend on the local generator mix, local load demands, and local market conditions, this paper will analyze one type of shift strategy to quantify the environmental benefit of shifting PEV charging to times when the grid is powered by cleaner power sources. The example will use California marginal grid emissions data, based on the model created in [17], shown in Figure 12.

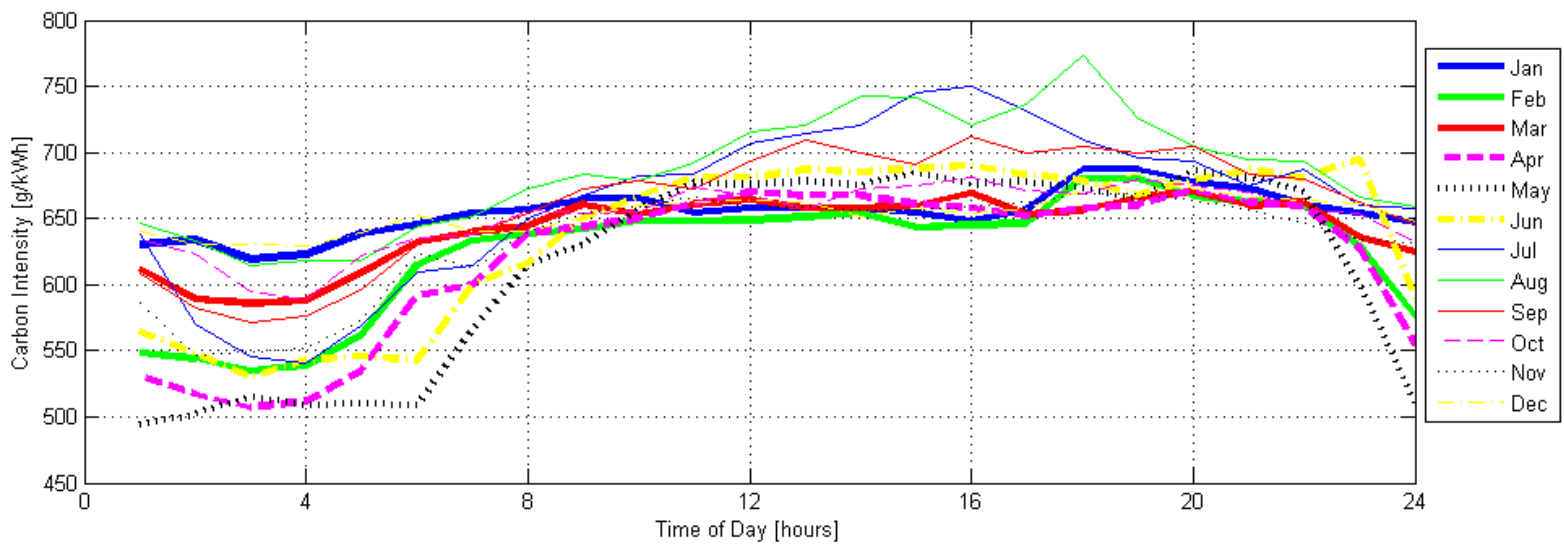

Figure 12: Estimated Marginal Carbon Intensity of Electricity Generation in California in 2010 
Based on the carbon intensity per kilowatt hour (CIPK) profile, the load shift strategy for this example was chosen to move all shiftable PEV charging load into the period between $11 \mathrm{pm}$ and $7 \mathrm{am}$. Shiftable load includes all PEV charging load that is not needed immediately by PEV drivers. The FleetCarma Charge Management Controller determines if charge is immediately needed based on vehicle state and user preferences. Thus, charge is considered immediately required if (i) the current vehicle SOC is less than the SOC Auto Opt-Out setting, or (ii) the 24Hr Opt-Out button has been hit in the last 24 hours, or (iii) charge is needed to ensure full battery charge is attained by the TCIN time setting. Therefore, this load shift analysis considers the actual load that can be shifted while not negatively affecting drivers

This analysis uses the October charging data from the ChargeTO pilot, as this is a mild-weather month, to determine the emissions savings possible given the California grid CIPK values. The carbon emission percentage savings are shown in Figure 13. These savings have been extrapolated to a pool of 1000 PEVs to find the monthly carbon emission savings, as shown in Figure 14. When the monthly savings are summed, the annual savings are over 110 metric tons. Since only shiftable load was moved in this analysis, these carbon reductions could be achieved with no negative impact on the driver.

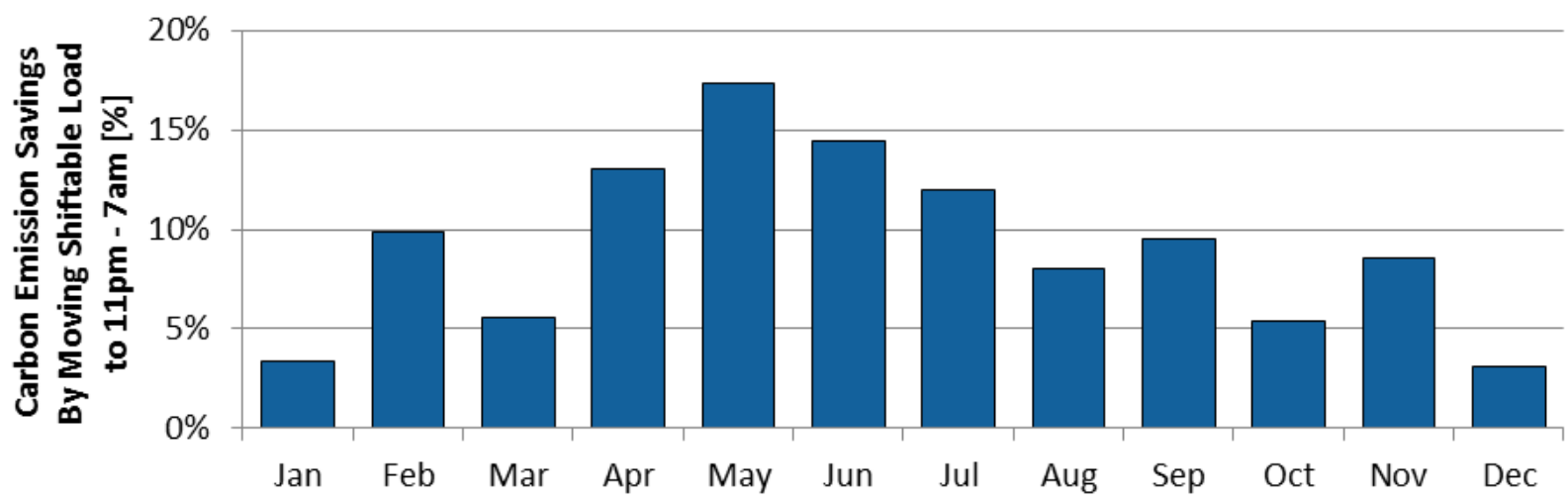

Figure 13: Potential PEV Charging Carbon Emission Reductions In California For 11pm to 7am Load Shift Strategy

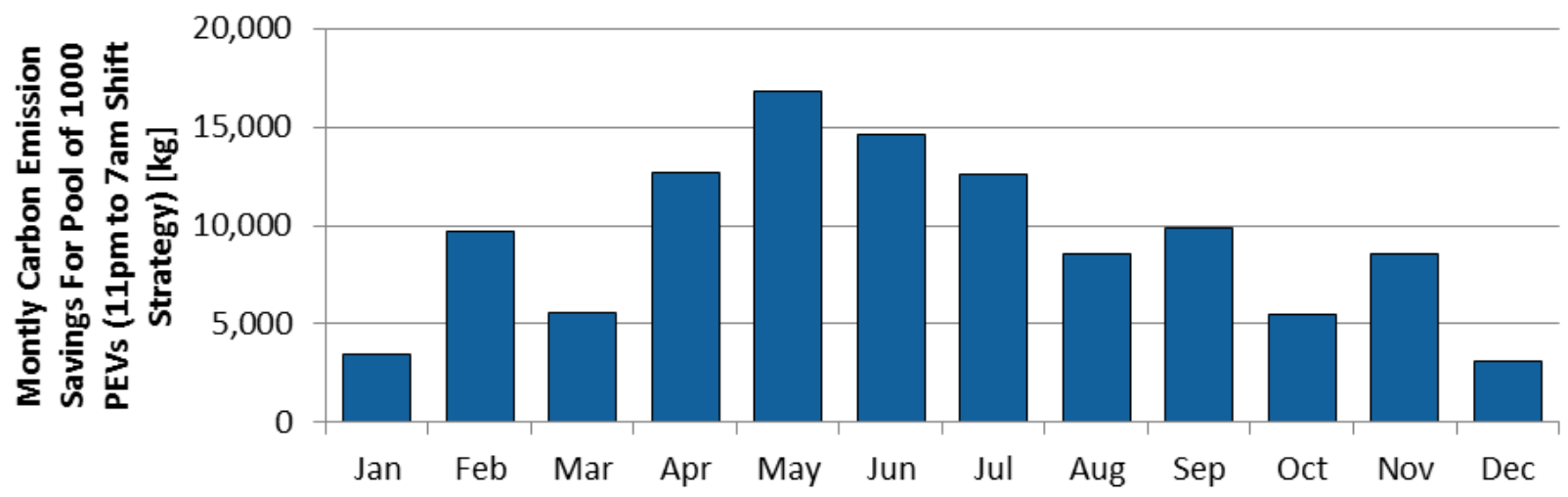

Figure 144: Potential Annual PEV Charging Carbon Emission Reductions In California For 11pm to 7am Load Shift Strategy For a Pool of 1000 PEVs

\section{Participant Learnings}

\subsection{Incentive Phase}

An incentive phase was run during October and November 2015. The goal was to determine if a reward could persuade the participants to make their vehicles more available for curtailment. The preference which was most often limiting charge curtailment up to this point in the program was the SOC Auto Opt-Out setting, because if people had set this preference to the maximum of $50 \%$, and they arrived home for the day below $50 \%$, their vehicle would require immediate charging at that time. Thus, the incentive was structured to encourage participants to lower this setting. The participants were divided into three groups: (1) a control group which received a set $\$ 40$ payment regardless of their settings, (2) a count-up incentive group, which 
started at $\$ 0$ and earned more reward for lowering their SOC Auto Opt-Out setting, and (3) a count-down group which started at $\$ 50$ and lost reward for having a higher SOC Auto Opt-Out setting.

Figure 5 shows that the incentive reward was definitely helpful in enticing participants to allow curtailments on their vehicle at lower battery SOCs. Whereas the majority of participants were at the $40-50 \%$ SOC setting prior to the incentive, the vast majority were in the $0-9 \%$ range after the incentive was offered. The incentive phase results are shown in Table 1. There was not a statistical difference between the behaviors of the countup group compared to the count-down group; both incentive methods worked equally well compared to the control group.

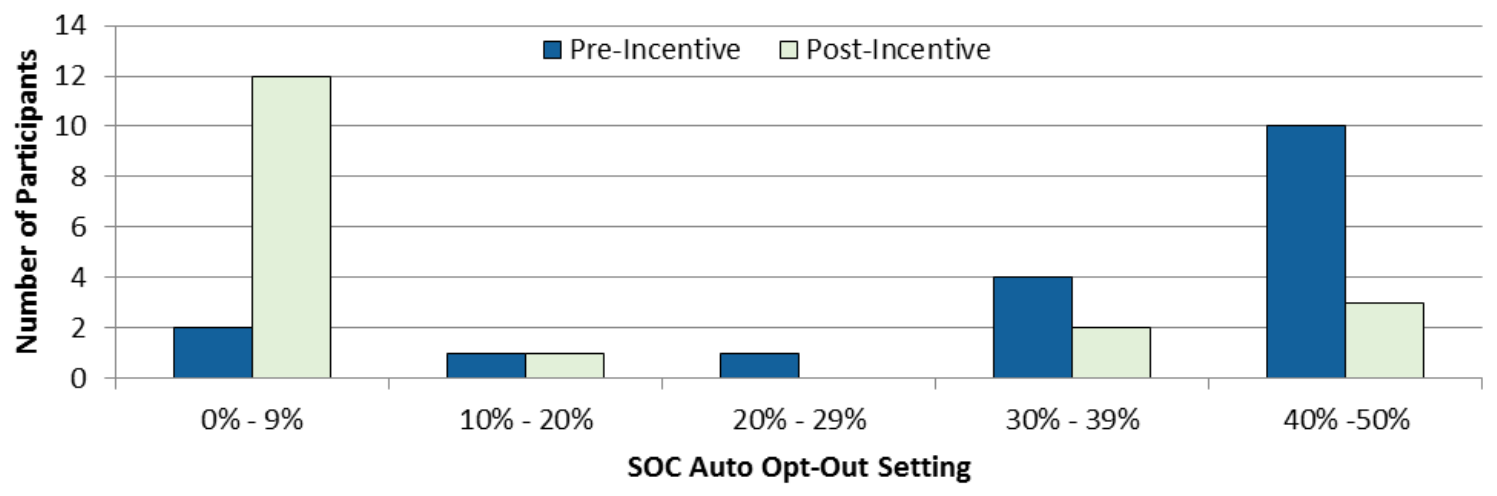

Figure 15: SOC Auto Opt-Out Thresholds for Incentivized Groups, Pre- and Post-Incentive

Table 1: Incentive Phase Results

\begin{tabular}{|l|c|c|c|}
\hline & Control Group & Count-Up Group & $\begin{array}{c}\text { Count-Down } \\
\text { Group }\end{array}$ \\
\hline Average SOC Auto Opt-Out Setting Pre-Incentive & $34 \%$ & $40 \%$ & $32.9 \%$ \\
\hline $\begin{array}{l}\text { Average SOC Auto Opt-Out Setting Post- } \\
\text { Incentive }\end{array}$ & $34 \%$ & $19 \%$ & $13.1 \%$ \\
\hline Average Incentive Paid Per Month & $\$ 20$ & $\$ 21.86$ & $\$ 22.97$ \\
\hline Cost Per Change in \% SOC & N/A & $\$ 1.04$ & $\$ 1.16$ \\
\hline
\end{tabular}

\subsection{Interviews}

Participant interviews were held at the start and end of the program. The participants gave positive feedback about the program, ranging from "An eye opener. It's fascinating. The portal is a wickedly powerful tool." to "Fairly invisible. It didn't impact me.". The "invisibility" of the charge curtailment was a common theme among participants, even though most vehicles were curtailed on most nights from August to December 2015. This is very encouraging feedback, as the goal of the system is to perform charge curtailments without impacting the driver's use of their vehicle.

Although many insights were obtained from the participants during the interviews, this paper will focus on two important questions, as shown in Figure6. The first question aimed to understand how important it was to participants that the smart-charging system considered their current battery SOC before performing any curtailment. A full $72 \%$ of the participants stated that they would not have signed up for the program if this critical vehicle-side data was not used in the curtailment algorithm. This is vitally important information in trying to develop a smart-charging program that has the potential for widespread adoption. The second question aimed to quantify the compensation required to for the participant to enroll in an ongoing smartcharging program with vehicle side data, without the free AddÉnergie EVSE and FleetCarma device that was provided in this particular pilot. The results reveal that $24 \%$ of participants would be interested in the program if they received no compensation other than access to their vehicle data, as was provided in this pilot. Over half of the participants would have allowed smart-charging of their vehicles with $\$ 10 /$ month or less of monetary incentive, with the participation rate rising to over $2 / 3$ rd with $\$ 15 /$ month or less of monetary incentive. 
Would you have signed up if your battery SOC
was not considered prior to curtailment?

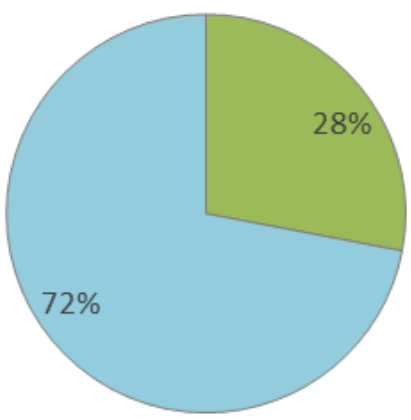

$\square$ Yes $\quad \square$ No
What would be adequate compensation for participating in Smart Charging?

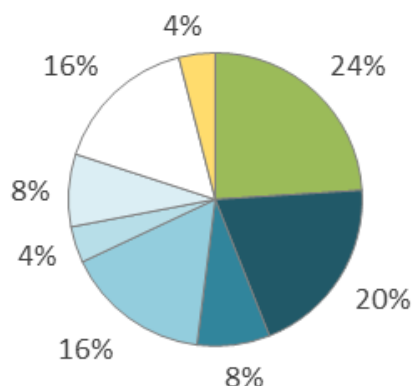

$\square$ Just Vehicle Data

$\square \$ 15 /$ month

$\square \$ 30+/$ month

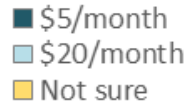

$\$ 10 /$ month $\square \$ 25 /$ month

Figure 16: Two Key Participant Interview Questions and Results

\section{Conclusions}

The ChargeTO pilot was the world's first known residential smart-charging demonstration to use vehicleside data on a variety of PEV models. Baseline charging data was captured over a 6-month period, which included categorization of charging behaviours into three archetypes. After the baseline data was captured, the was actively managed over a 5 -month period. During the 150 -day period the majority of the participants were curtailed nightly. The curtailment objectives were varied to simulate a number of potential grid scenarios and resulting demand response priorities. At peak load times $70-80 \%$ of the charging load could be shed (instantaneous curtailment capacity) while still ensuring all vehicles were fully charged by their preset departure time. Interview results indicated that $72 \%$ of participants would require the use of vehicle-side data in any future smart-charging program. These results indicate that vehicle-side data will be a critical component of any smart-charging program that will achieve widespread adoption.

\section{Acknowledgments}

The authors wish to thank the Ontario Ministry of Energy and the program participants.

\section{References}

[1] (accessed 2016-03-23) http://gm-volt.com/2015/09/16/world-crosses-one-million-plug-in-sales-milestone/

[2] (accessed 2016-03-23) http://www.bloomberg.com/features/2016-ev-oil-crisis/

[3] K. Qian, C. Zhou, M. Allan, Y. Yuan, Modeling of Load Demand Due to EV Battery Charging in Distribution Systems, IEEE Transactions on Power Systems, Vol. 26, Issue 2, p. 802-810, August 2010.

[4] (accessed 2016-03-23) https://www.technologyreview.com/s/518066/could-electric-cars-threaten-the-grid/

[5] R. A. Verzijlbergh, M. O. W. Grond, Z. Lukszo, j.G. Slootweg, M. D. Ilic, Network Impacts and Cost Savings of Controlled EV Charging, IEEE Transactions on Smart Grid, Vol. 3, Issue 3, p. 1203 - 1212, May 2012.

[6] J. Bailey, J. Axsen, Anticipating PEV buyers' acceptance of utility controlled charging, Transportation Research Part A. Vol. 82, p. 29-46, December 2015.

[7] H. Makkonen, V. Tikka, J. Lassila, J. Partanen, P. Silventoinen, Demonstration of Smart Charging Interface in Green Campus, $201416^{\text {th }}$ European Conference on Power Electronics and Applications, p. 1-10, August 26-28, 2014.

[8] Pilot Project with IBM, Honda, and PG\&E (accessed 2016-03-23) http://www03.ibm.com/press/us/en/pressrelease/37398.wss

[9] FlexPower Project by ElaadNL (accessed 2016-03-23) http://elaad.nl/flexpower/

[10] Demand Response in Workplace Charging Project by Southern California Edison (accessed 2016-03-23) http://www.prnewswire.com/news-releases/greenlots-supports-southern-california-edisons-deployment-of-80$\underline{\text { level-2-chargers-at-multiple-sites-for-demand-response-in-workplace-charging-300035520.html }}$ 
[11] T. Shimizu, A. Yokoyama, K. Sato, K. Kumita, Experimental Demonstration of Smart Charging and Demand Response for Plug-in Electric Vehicles Based on SAE Standards, SAE Technical Paper 2015-01-0301, 2015.

[12] My Electric Avenue Project Summary Report (accessed 2016-03-23) http://myelectricavenue.info/sites/default/files/My\%20Electric\%20Avenue\%20\%28I2EV\%29\%20\%20Project\%20Summary\%20Report.pdf

[13] Jump Smart Maui Project with Nissan (accessed 2016-03-23) http://www.jumpsmartmaui.com/jumpsmartmauiseeking-volunteers-phase-2-smartpower/

[14] BMW i Chargeforward Project with PG\&E (accessed 2016-03-23) http://www.bmwichargeforward.com/

[15] California ISO, Demand Response and Energy Efficiency Roadmap: Maximizing Preferred Resources, December 2013. (accessed 2016-03-23) https://www.caiso.com/Documents/DR-EERoadmap.pdf

[16] P. Denholm, M. O’Connell, G. Brinkman, J. Jorgenson, Overgeneration from Solar Energy in California: A Field Guide to the Duck Chart, National Renewable Energy Laboratory Technical Report, November 2015. (accessed 2016-03-23) http://www.nrel.gov/docs/fy16osti/65023.pdf

[17] R. McCarthy, C. Yang, Determining Marginal Electricity For Near-Term Plug-In and Fuel Cell Vehicle Demands in California: Impacts on Vehicle Greenhouse Gas Emissions, Journal of Power Sources, 2009, doi: 10.1016/jpowsour.2009.10.024.

\section{Authors}
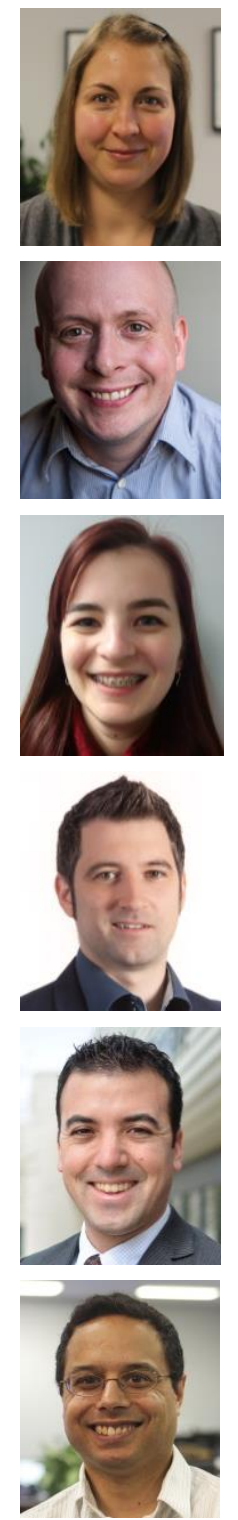

Jennifer Bauman, PhD, PEng, is the Director of Research at FleetCarma. Jennifer has extensive experience in vehicle architecture design and optimization, as well as analysis, simulation, and problem-solving with respect to the real-world issues faced by the next generation of advanced vehicles. Jennifer holds a Ph.D. in electrical engineering from the University of Waterloo. Her Ph.D. research focused on power electronics for hybrid electric vehicles, and simulation and optimization of fuel cell vehicles.

Matthew Stevens, PhD, has been the Chief Executive Officer of FleetCarma since 2009. Matt sits on the board of Electric Mobility Canada (EMC) and is an adjunct professor at the University of Waterloo. Prior to FleetCarma, Matt held technical roles at BlackBerry and Nordion. Matt holds a PhD in chemical engineering from the University of Waterloo. His research interests include hybrid vehicle control, battery state-of-health, and smart-charging of plug-in vehicles.

Simone Hacikyan, BSc, is a Customer Success and Project Manager at FleetCarma, where she focuses on streamlining processes to improve the experience of FleetCarma clients. In the ChargeTO pilot, Simone was involved the day-to-day project execution, including system testing and troubleshooting, and participant inteviews. Simone recevied the B.Sc. degree in Science and Business from the University of Waterloo.

Louis Tremblay, MASc, is the President, CEO, and cofounder of AddÉnergie. AddÉnergie is a leading Canadian smart charging solutions provider for electric vehicles, with more than 2,000 charging stations operating throughout the country. Louis graduated from Laval University with a Bachelor's Degree in Electrical Engineering and a Master's Degree in Power electronics. He is also a Board member of Electric Mobility Canada (EMC) and the Innovative Vehicle Institute (IVI).

Eric Mallia, MES, is General Manager at FleetCarma and is a contributing author in the book Electric Vehicle Business Models - Global Perspectives. His international work with more than 150 organizations has focused on the implementation of efficiency programs in transportation, particularly with the adoption and proliferation of plug-in vehicles. Eric holds a Master's degree in Environmental Studies from the University of Waterloo and a Bachelor's degree in Business Administration from Wilfrid Laurier University.

Chris Mendes, MASc, is FleetCarma's Chief Technology Officer and is responsible for developing FleetCarma's technology portfolio and defining the technology product strategy. Prior to FleetCarma, Chris was involved in advanced motor development and control system development. He earned a MASc in mechanical engineering from the University of Waterloo, where his research focused on hybrid vehicle design and advanced torque control strategies. In 2007, he co-founded FleetCarma. 